\title{
Rikkunshito, a traditional Japanese medicine, suppresses cisplatin-induced anorexia in humans
}

This article was published in the following Dove Press journal:

Clinical and Experimental Gastroenterology

9 December 201 I

Number of times this article has been viewed

\section{Tetsuro Ohno \\ Mitsuhiro Yanai \\ Hiroyuki Ando \\ Yoshitaka Toyomasu \\ Atsushi Ogawa \\ Hiroki Morita \\ Kyoichi Ogata \\ Erito Mochiki \\ Takayuki Asao \\ Hiroyuki Kuwano}

Department of General Surgical Science, Gunma University Graduate School of Medicine, Maebashi, Japan

Correspondence: Tetsuro Ohno

Department of General Surgical Science,

Gunma University Graduate School

of Medicine, 3-39-22 Showa-machi,

Maebashi 37I-85II, Japan

$\mathrm{Tel}+8 \mathrm{I} 272208224$

Fax $+8 \mid 272208230$

Email tetsuro@med.gunma-u.ac.jp
Background: The aim of this study was to investigate the effects of Rikkunshito on ghrelin secretion and on cisplatin-induced anorexia in humans.

Methods: The study was performed as a crossover design, and ten unresectable or relapsed gastric cancer patients were randomly divided into two groups. Group A ( $\mathrm{n}=5)$ was started on Rikkunshito (2.5 $\mathrm{g}$ three times daily, orally) from the first course of chemotherapy and followed by a second course without Rikkunshito. A treatment with reversed order was performed for Group B $(\mathrm{n}=5)$. All patients received combined chemotherapy with S-1 plus cisplatin. The primary endpoint was the amount of oral intake, and the categories of scales of anorexia, nausea, and vomiting; secondary endpoints included the plasma concentration of acylated ghrelin.

Results: In the Rikkunshito-on period, no decrease of the plasma concentration of acylated ghrelin induced by cisplatin was observed. The average oral intake in the Rikkunshito-on period was significantly larger than that in the Rikkunshito-off period, and the grade of anorexia was significantly lower in the Rikkunshito-on period than in the Rikkunshito-off period.

Conclusion: Rikkunshito appeared to prevent anorexia induced by cisplatin, resulting in effective prophylactic administration of chemotherapy with cisplatin, and patients could continue their treatments on schedule.

Keywords: Rikkunshito, cisplatin, ghrelin, anorexia, stomach cancer

\section{Introduction}

Combined chemotherapy with $\mathrm{S}-1$ plus cisplatin is an attractive chemotherapy regimen for gastric cancer. A previous Phase I/II trial of this regimen in metastatic gastric cancer reported a high response rate of $76 \%$ and acceptable toxicity. ${ }^{1}$ Recently, a Phase III Japanese trial of chemotherapeutic regimens for metastatic gastric cancer (SPIRITS trial) demonstrated that S-1 plus cisplatin led to significantly longer median overall survival than S-1 alone (13 months versus 11 months). ${ }^{2}$ Cisplatin is widely used in various chemotherapies, but undesirable side effects, such as nausea, vomiting, and anorexia, markedly affect the quality of life of patients and may make the continuation of chemotherapy difficult. While some antiemetic agents have been introduced as treatment for nausea and vomiting, ${ }^{3,4}$ appetite loss is still present in many cancer patients. However, the mechanism of the resulting appetite loss during chemotherapy is not thoroughly understood.

Ghrelin is an endogenous ligand of the growth hormone secretagogue receptor; it consists of 28 amino acids and is secreted mainly from the stomach. ${ }^{5}$ Ghrelin is known to have an intense appetite-enhancing effect in addition to a growth hormone secretion-promoting effect. ${ }^{6}$ Ghrelin is the only hormone that exhibits an orexigenic 
effect following peripheral administration. ${ }^{7}$ In addition, ghrelin exhibits a variety of actions, including stimulation of growth hormone secretion, gastric motility, and gastric acid secretion, ${ }^{8}$ as well as induction of a positive energy balance. ${ }^{9}$ The level of plasma ghrelin is thought to be related to gastrointestinal disorders, and ghrelin has been administered to patients with anorexia-related disorders as a new therapy. ${ }^{10}$ However, the intravenous and repeated administration of ghrelin presented a considerable burden for the patients.

Rikkunshito, a traditional Japanese medicine, is used to treat various gastrointestinal tract disorders, such as functional dyspepsia, gastroesophageal reflux, dyspeptic symptoms of post-gastrointestinal surgery, and chemotherapy-induced nausea. ${ }^{1-13}$ Takeda et $\mathrm{al}^{14}$ showed that a flavonoid in Rikkunshito suppressed a cisplatin-induced decrease in plasma acylated ghrelin levels and increased food intake in rats, and was mediated by serotonin $\left(5-\mathrm{HT}_{2 \mathrm{~B}}\right.$ and $\left.5-\mathrm{HT}_{2 \mathrm{C}}\right)$ receptors. The aim of this study was to investigate the effect of Rikkunshito on ghrelin secretion and on cisplatin-induced anorexia in humans.

\section{Materials and methods Patient eligibility}

Eligible patients had histologically proved unresectable or recurrent gastric cancer. Up to one regimen of prior chemotherapy was allowed (adjuvant chemotherapy was allowed provided that at least 28 days had elapsed since the last treatment), except for prior treatment with cisplatin.

Other inclusion criteria were as follows: age 20-75 years; Eastern Clinical Oncology Group performance status 0-1; estimated life expectancy more than 3 months; a white blood cell count between 4000 and $12,000 \mathrm{~mm}^{3}$; an absolute neutrophil count of over $2000 \mathrm{~mm}^{3}$, a platelet count of over
$100,000 \mathrm{~mm}^{3}$, and a hemoglobin level of over $8.0 \mathrm{~g} / \mathrm{dL}$; aspartate aminotransferase and alanine aminotransferase levels within two times the upper limit of normal for the institution; serum bilirubin level less than $1.5 \mathrm{mg} / \mathrm{dL}$; serum creatinine level within the upper limit of the normal value for the institution; 24-hour creatinine clearance more than $50 \mathrm{~mL} /$ minute; and a normal electrocardiogram. Only patients who could swallow tablets were eligible. Patients were excluded if they had brain metastases, severe comorbid conditions, active double cancers, or a past history of drug allergy or were unable to comply with the protocol requirements. Pregnant women were also excluded. Written informed consent was obtained from all patients before study entry.

\section{Treatment design}

The study was performed as a crossover design because of the limited number of patients (Figure 1). Ten patients were randomly divided into two groups. The gender, age, tumor characteristics, and performance status of these 10 patients are listed in Table 1. Group A $(n=5)$ was started on Rikkunshito from the first course of chemotherapy followed by a second course of chemotherapy without Rikkunshito. Treatment with reversed order was performed for Group B $(n=5)$. Patients in the intervention period took Rikkunshito (Tsumura Co, Ltd, Tokyo, Japan) at a daily dose of $7.5 \mathrm{~g}$ (2.5 $\mathrm{g}$ three times daily, orally) before every meal through chemotherapy from days 1 to 21. On the other hand, patients in the control period took nothing. All patients received combined chemotherapy with S-1 plus cisplatin as follows. S-1 (Taiho Pharmaceutical Co, Ltd, Tokyo, Japan) was given orally twice daily after meals at a fixed dose of $80 \mathrm{mg} / \mathrm{m}^{2} /$ day for 21 consecutive days followed by a 14-day rest period; this cycle was repeated every 5 weeks. The dose of S-1,

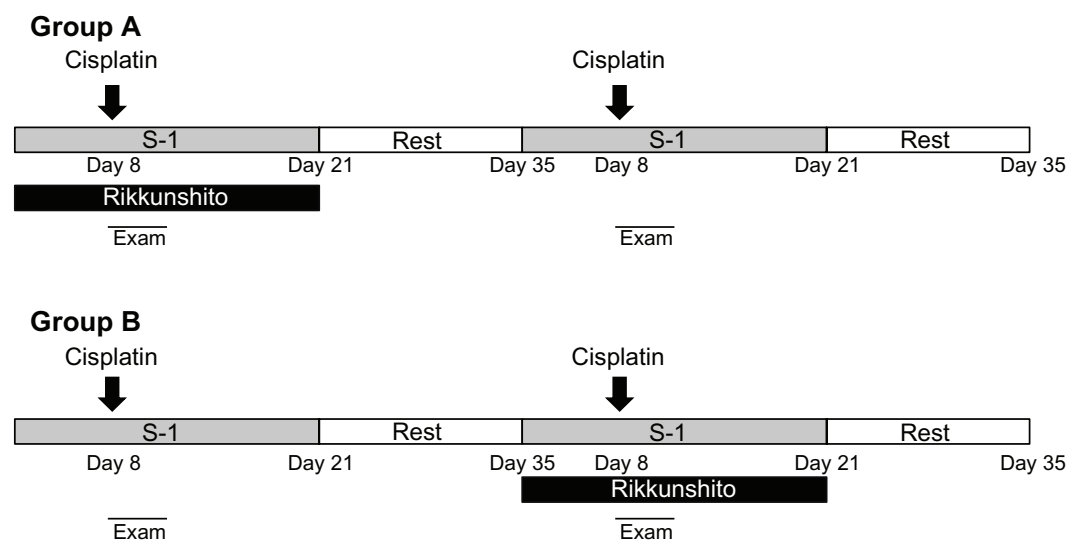

Figure I Crossover study design. Patients in group A initially took oral Rikkunshito before every meal for 3 weeks (on treatment). After a rest period of 2 weeks, Rikkunshito was discontinued for 3 weeks (off treatment). Conversely, patients in group B initially were off treatment for 3 weeks and then on treatment for 3 weeks after the rest period. 
Table I Background of patients

\begin{tabular}{llllll}
\hline Patient & Age (years) & Sex & Tumor & PS & Group \\
\hline I & 55 & M & Recurrent & I & A \\
2 & $6 I$ & F & Unresectable & 0 & A \\
3 & 67 & M & Recurrent & 0 & B \\
4 & 71 & M & Unresectable & 0 & A \\
5 & 52 & M & Unresectable & I & A \\
6 & 72 & M & Recurrent & 0 & B \\
7 & 50 & F & Unresectable & I & B \\
8 & 62 & M & Unresectable & 0 & B \\
9 & 67 & M & Recurrent & I & A \\
10 & $6 I$ & M & Unresectable & 0 & B \\
\hline
\end{tabular}

Abbreviation: PS, performance status.

decided on the basis of body surface area (BSA), was $80 \mathrm{mg}$ $\left(\right.$ BSA $<1.25 \mathrm{~m}^{2}$ ), $100 \mathrm{mg}$ (BSA $1.25-1.5 \mathrm{~m}^{2}$ ), or $120 \mathrm{mg}$ $\left(\mathrm{BSA} \geq 1.5 \mathrm{~m}^{2}\right)$. Cisplatin (Landa ${ }^{\mathrm{TM}}$, Nippon Kayaku Co, Ltd, Tokyo, Japan) was administered intravenously on day 8 . All patients received $16 \mathrm{mg}$ of dexamethasone and $3 \mathrm{mg}$ of granisetron intravenously one hour before cisplatin infusion and $8 \mathrm{mg}$ of dexamethasone on days 9 and 10 . The initially administered dose of cisplatin was $60 \mathrm{mg} / \mathrm{m}^{2}$. Blood samples were obtained from each patient before the administration of cisplatin on day 8 and 3 hours after the administration of cisplatin was finished. The primary endpoint was the amount of oral intake, and the categories of the scales of anorexia, nausea, and vomiting; secondary endpoints included the plasma concentration of acylated ghrelin.

\section{Measurement of acylated ghrelin}

The blood samples were collected before and 3 hours after the administration of cisplatin. The sample collecting time was determined on the basis of our unpublished experimental data from dogs. The plasma samples for acylated ghrelin were promptly centrifuged at $4{ }^{\circ} \mathrm{C}$, and the supernatants were acidified with $1 \mathrm{~mol} / \mathrm{L} \mathrm{HCl}$ (1/10 volume), frozen, and kept below $40^{\circ} \mathrm{C}$ until measurement. The acylated ghrelin level was determined using the active ghrelin enzyme-linked immunosorbent assay kit (SCETI Co, Ltd, Tokyo, Japan).

\section{Definition of response}

Following administration of cisplatin, each patient was hospitalized and monitored by direct observation and patient interview for 5 days. The amount of oral intake of each meal was observed and scored by 11 stages from 0 to 10 by nurses, and the average oral intake during 5 days was calculated and analyzed. Categories of the scales for anorexia, nausea, and vomiting were graded according to the National Cancer Institute common toxicity criteria, version $3.0 .{ }^{15} \mathrm{We}$ defined the time to treatment failure as the period between the time that administration of cisplatin was finished and the time that vomiting or dry vomiting occurred or the time of administration of the antiemetic.

\section{Statistical analysis}

The results were expressed as the mean \pm the standard error of the mean. The Student's $t$-test was used to test for the significance of differences between groups. For the comparison of time to treatment failure between two groups, the KaplanMeier product-limit method and log-rank test were used. A $P$ value $<0.05$ was considered statistically significant. Statistical calculations were performed using StatView ${ }^{\circledR}$ software (version 5.0, Abacus Concepts Inc, Berkeley, CA).

\section{Results \\ Plasma concentration of acylated ghrelin}

In the Rikkunshito-off period, the average concentration of plasma acylated ghrelin 3 hours after the administration of cisplatin decreased from that before administration of cisplatin, but the difference was not significant. On the other hand, in the Rikkunshito-on period, no decrease in plasma concentration of acylated ghrelin was observed between before and after administration (Figure 2).

\section{Amount of oral intake}

The average oral intake in the Rikkunshito-on period was significantly larger than that in the Rikkunshito-off period (6.29 versus 3.94, $P=0.0496$, Figure 3 ). This tendency was similarly seen in group A and group B, and neither an order effect nor a carry-over effect was seen.

\section{Anorexia, nausea, vomiting}

The grade of anorexia (0-4) was significantly lower in the Rikkunshito-on period than in the Rikkunshito-off period

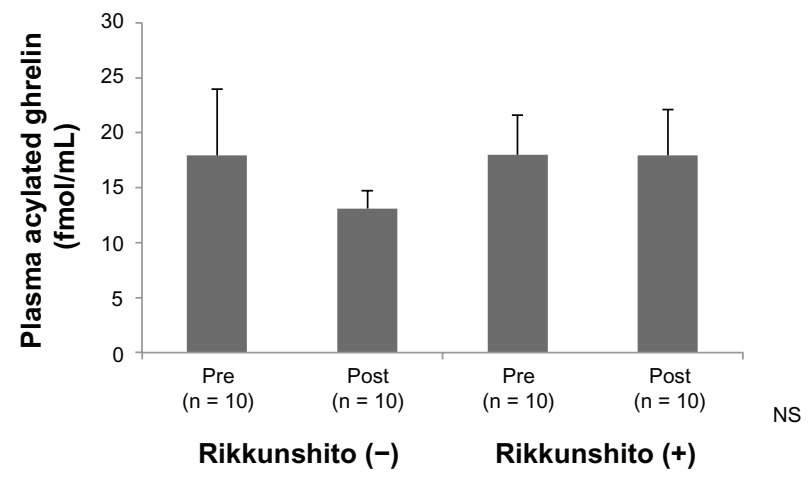

Figure 2 Plasma concentration of acylated ghrelin. In the Rikkunshito-on period (Rikkunshito $[+]$ ), no decrease of plasma concentration of acylated ghrelin was observed before and after administration.

Abbreviation: NS, not significant. 


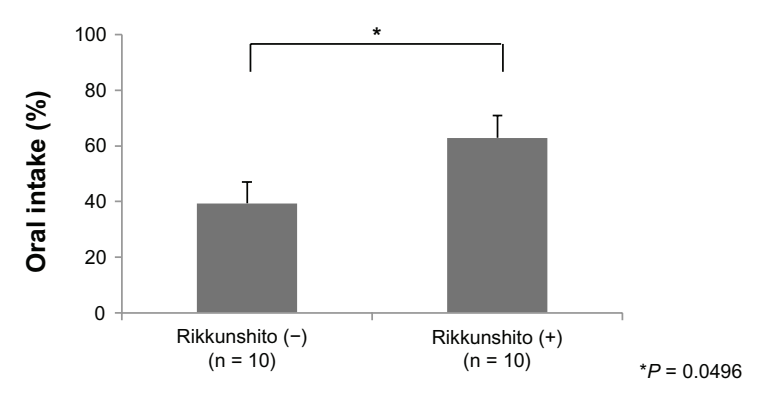

Figure 3 Comparison of the amount of oral intake. The average oral intake in the Rikkunshito-on period was significantly larger than that in the Rikkunshito-off period. Note: $* P=0.0496$.

(1.2 versus $2.2, P=0.0441$, Figure 4 ). No order effect or carryover effect was seen. The difference in the grade of nausea $(0-3)$ was not significant, although the grade in the Rikkunshito-on period tended to be lower (Figure 5). The difference in grade of vomiting (0-4) was not significant (Figure 6).

\section{Time to treatment failure}

The number of cases of treatment failure in the Rikkunshitooff period was nine, whereas that in the Rikkunshito-on period was five. However, the difference was not significant (Figure 7).

\section{Toxicity}

Pseudohyperaldosteronism and hepatic toxicity have been reported as side effects of Rikkunshito. We assessed hypertension, edema, hypokalemia, and transaminitis. These events did not appear for either the Rikkunshito-on or the Rikkunshito-off period, and all patients were able to complete the examination.

\section{Discussion}

Ghrelin has been measured in patients with various diseases, for example, functional dyspepsia, ${ }^{16}$ breast cancer, colon cancer, ${ }^{17}$ and hepatocellular carcinoma, ${ }^{18}$ and the relationship between the disease and the level of ghrelin has

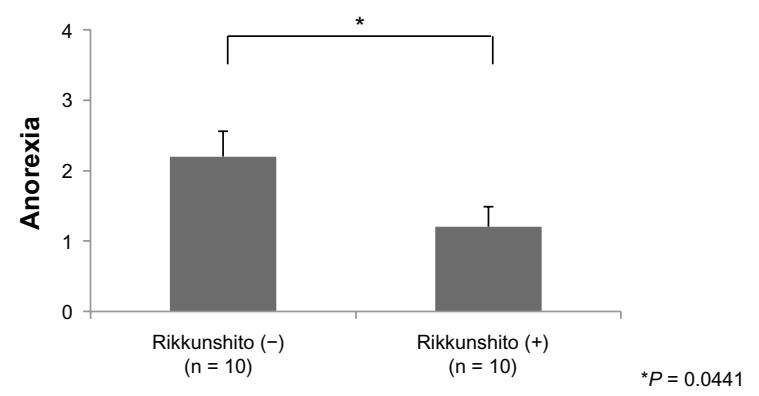

Figure 4 Comparison of the grade of anorexia. The grade of anorexia (0-4) was significantly lower in the Rikkunshito-on period than in the Rikkunshito-off period. Note: $* P=0.0441$.

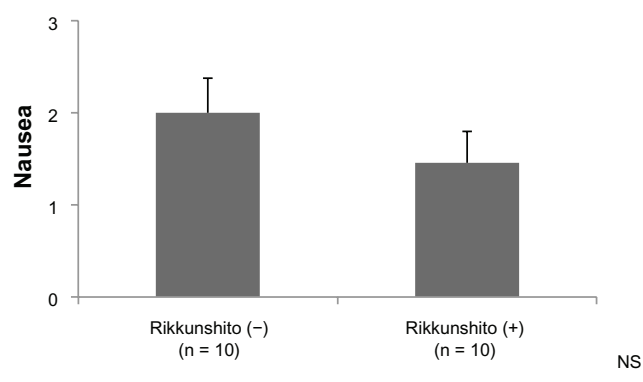

Figure 5 Comparison of the grade of nausea. The difference in the grade of nausea (0-3) was not significant.

Abbreviation: NS, not significant.

been assessed. Garcia et $\mathrm{al}^{19}$ reported that plasma ghrelin levels were higher in cachectic patients than in noncachectic ones, suggesting that an increase in the plasma ghrelin level might be related to secondary loss of appetite in patients. The plasma ghrelin level in patients with obesity was low, and the decrease in body weight was proportional to increasing ghrelin levels. ${ }^{20}$ The ghrelin level of patients with anorexia nervosa was reported to be high. ${ }^{21}$ It remains controversial whether the plasma ghrelin level of patients with functional dyspepsia increases or decreases. ${ }^{22,23}$ Akamizu et al ${ }^{24}$ experimented with the administration of ghrelin in patients with functional dyspepsia; their results showed that daily food intake tended to increase in comparison with intake before and after completion of treatment. However, the difference was not significant. The hunger sensation was reported to be significantly elevated. It is certain that ghrelin is related to appetite; however, the manner in which it affects appetite and food intake remains unclear.

Rikkunshito is a traditional Japanese medicine (Kampo) that is widely used for treating upper gastrointestinal symptoms, such as decreased gastric motility after surgery ${ }^{11}$ and chronic idiopathic dyspepsia, ${ }^{12}$ and its beneficial effects have been shown. Rikkunshito contains mixed raw herbs in the following ratios: JP Atractylodes lancea rhizome 4.0 g, JP Ginseng 4.0 g, JP Pinellia tuber 4.0 g, JP Poria

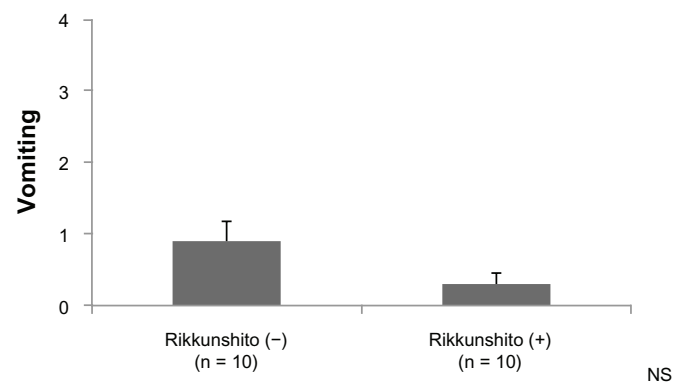

Figure 6 Comparison of the grade of vomiting. The difference in the grade of vomiting (0-4) was not significant.

Abbreviation: NS, not significant. 


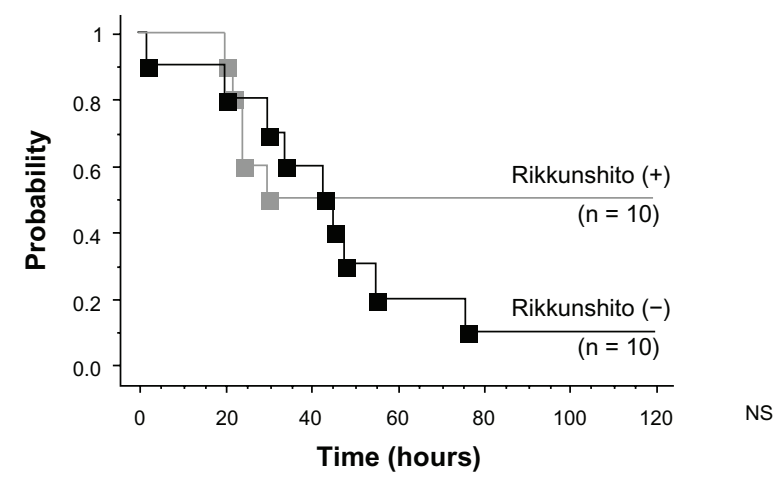

Figure 7 Kaplan-Meier curves of time to treatment failure. The number of cases of treatment failure in the Rikkunshito-off period was nine, whereas that in the Rikkunshito-on period was five.

Abbreviation: NS, not significant.

sclerotium $4.0 \mathrm{~g}$, JP Jujube $2.0 \mathrm{~g}$, JP Citrus unshiu peel $2.0 \mathrm{~g}$, $J P$ Glycyrrhiza $1.0 \mathrm{~g}$, and JP Ginger $0.5 \mathrm{~g}$. Rikkunshito has been approved for medicinal use by the Japanese Ministry of Health and Welfare, although the mechanism by which Rikkunshito alleviates upper gastrointestinal symptoms has not been clarified. Recently, Rikkunshito was used in combination with granisetron to alleviate the side effects of anticancer drugs, without affecting their efficacy. ${ }^{12}$ Decreased gastric motility and decreased appetite as a result of surgery, anticancer drugs, and functional dyspepsia may be caused by a decreased plasma ghrelin level ${ }^{25,26}$ or ghrelin function. In clinical applications, Rikkunshito is particularly effective against functional dyspepsia, ${ }^{11}$ and its efficacy is also supported by basic research in rats with a delayed gastric emptying function. ${ }^{27}$ Matsumura et $\mathrm{al}^{28}$ reported that Rikkunshito significantly increased the plasma acylated ghrelin level in healthy volunteers and normal mice, and the ghrelin mRNA expression level in the stomach was upregulated in mice. These findings suggest the possibility that Rikkunshito affects the secretion or function of ghrelin.

Takeda et $\mathrm{al}^{14}$ reported that Rikkunshito suppresses a cisplatin-induced decrease in the plasma level of ghrelin, a hormone that stimulates gastrointestinal motility and food intake in rats. Heptomethoxyflavone, hesperidin, and isoliquiritigenin, the flavonoids in Rikkunshito, are reported to be responsible for a $5 \mathrm{HT}_{2 \mathrm{~B}}$ antagonistic effect and restoration of the plasma level of ghrelin.

In the present study, in the Rikkunshito-off period, the average concentration of plasma acylated ghrelin after administration decreased from that before administration, but the difference was not significant. On the other hand, in the Rikkunshito-on period, a decrease of plasma concentration of acylated ghrelin was not observed before nor after administration (Figure 2). This result does not contradict the findings of Takeda et al. Rikkunshito might restore the plasma level of ghrelin.

The average oral intake in the Rikkunshito-on period was significantly larger than that in the Rikkunshito-off period (Figure 3), and the grade of anorexia was significantly lower in the Rikkunshito-on period than that in the Rikkunshito-off period (Figure 4). These results might also indicate the effect of restoration of ghrelin. On the other hand, the differences in the grade of nausea, vomiting, and time to treatment failure between the two groups were not significant (Figures 5, 6, and 7). Nausea and vomiting are reactions mainly related to $5 \mathrm{HT}_{3}$ receptors, and the relationship with ghrelin might be minimal.

In summary, Rikkunshito might prevent anorexia induced by cisplatin; thus, prophylactic administration was effective in chemotherapy with cisplatin, and patients could continue their treatment on schedule. This is the first report demonstrating the beneficial effects of Rikkunshito in the treatment of cisplatin therapy in humans. A limitation of the present study is the small number of patients. It will be necessary to confirm the usefulness of Rikkunshito by performing larger randomized controlled studies in the future.

\section{Disclosure}

The authors report no conflicts of interest in this work.

\section{References}

1. Koizumi W, Tanabe S, Saigenji K, et al. Phase I/II study of S-1 combined with cisplatin in patients with advanced gastric cancer. $\mathrm{Br} J$ Cancer. 2003;89(12):2207-2212.

2. Koizumi W, Narahara H, Hara T, et al. S-1 plus cisplatin versus S-1 alone for first-line treatment of advanced gastric cancer (SPIRITS trial): a phase III trial. Lancet Oncol. 2008;9(3):215-221.

3. Longo F, Mansueto G, Lapadula V, et al. Palonosetron plus 3-day aprepitant and dexamethasone to prevent nausea and vomiting in patients receiving highly emetogenic chemotherapy. Support Care Cancer. 2011; 19(8):1159-1164

4. Aapro M, Fabi A, Nole F, et al. Double-blind, randomised, controlled study of the efficacy and tolerability of palonosetron plus dexamethasone for 1 day with or without dexamethasone on days 2 and 3 in the prevention of nausea and vomiting induced by moderately emetogenic chemotherapy. Ann Oncol. 2010;21(5):1083-1088.

5. Kojima M, Hosoda H, Date Y, Nakazato M, Matsuo H, Kangawa K. Ghrelin is a growth-hormone-releasing acylated peptide from stomach. Nature. 1999;402(6762):656-660.

6. Nakazato M, Murakami N, Date Y, et al. A role for ghrelin in the central regulation of feeding. Nature. 2001;409(6817):194-198.

7. Wren AM, Seal LJ, Cohen MA, et al. Ghrelin enhances appetite and increases food intake in humans. J Clin Endocrinol Metab. 2001;86(12): 5992.

8. Masuda Y, Tanaka T, Inomata N, et al. Ghrelin stimulates gastric acid secretion and motility in rats. Biochem Biophys Res Commun. 2000; 276(3):905-908.

9. Asakawa A, Inui A, Kaga T, et al. Antagonism of ghrelin receptor reduces food intake and body weight gain in mice. Gut. 2003;52(7):947-952. 
10. Hotta M, Ohwada R, Akamizu T, Shibasaki T, Takano K, Kangawa K. Ghrelin increases hunger and food intake in patients with restricting-type anorexia nervosa: a pilot study. Endocr J. 2009;56(9): 1119-1128.

11. Tatsuta M, Iishi H. Effect of treatment with liu-jun-zi-tang (TJ-43) on gastric emptying and gastrointestinal symptoms in dyspeptic patients. Aliment Pharmacol Ther. 1993;7(4):459-462.

12. Tomono H, Ito Y, Watanabe T. Successful antiemetic treatment of TSUMURA Rikkunshi-to Extract Granules for ethical use in addition to other antiemetic agents in neoadjuvant chemotherapy for an advanced breast cancer patient. Gan To Kagaku Ryoho. 2006;33(8):1129-1131. Japanese.

13. Oka T, Tamagawa Y, Hayashida S, Kaneda Y, Kodama N, Tsuji S. Rikkunshi-to attenuates adverse gastrointestinal symptoms induced by fluvoxamine. Biopsychosoc Med. 2007;1:21.

14. Takeda H, Sadakane C, Hattori T, et al. Rikkunshito, an herbal medicine, suppresses cisplatin-induced anorexia in rats via $5-\mathrm{HT}_{2}$ receptor antagonism. Gastroenterology. 2008;134(7):2004-2013.

15. Japanese translation of common terminology criteria for adverse events (CTCAE), and instructions and guidelines. Int J Clin Oncol. 2004;9 Suppl 3:1-82.

16. Shinomiya T, Fukunaga M, Akamizu T, et al. Plasma acylated ghrelin levels correlate with subjective symptoms of functional dyspepsia in female patients. Scand J Gastroenterol. 2005;40(6):648-653.

17. Wolf I, Sadetzki S, Kanety H, et al. Adiponectin, ghrelin, and leptin in cancer cachexia in breast and colon cancer patients. Cancer. 2006; 106(4):966-973.

18. Ataseven H, Bahcecioglu IH, Kuzu N, et al. The levels of ghrelin, leptin, TNF-alpha, and IL-6 in liver cirrhosis and hepatocellular carcinoma due to HBV and HDV infection. Mediators Inflamm. 2006; 2006(4):78380.
19. Garcia JM, Garcia-Touza M, Hijazi RA, et al. Active ghrelin levels and active to total ghrelin ratio in cancer-induced cachexia. J Clin Endocrinol Metab. 2005;90(5):2920-2926.

20. Soriano-Guillen L, Barrios V, Campos-Barros A, Argente J. Ghrelin levels in obesity and anorexia nervosa: effect of weight reduction or recuperation. J Pediatr. 2004;144(1):36-42.

21. Dostalova I, Haluzik M. The role of ghrelin in the regulation of food intake in patients with obesity and anorexia nervosa. Physiol Res. 2009;58(2):159-170.

22. Takamori K, Mizuta Y, Takeshima F, et al. Relation among plasma ghrelin level, gastric emptying, and psychologic condition in patients with functional dyspepsia. J Clin Gastroenterol. 2007;41(5):477-483.

23. Pilichiewicz AN, Feltrin KL, Horowitz M, et al. Functional dyspepsia is associated with a greater symptomatic response to fat but not carbohydrate, increased fasting and postprandial CCK, and diminished PYY. Am J Gastroenterol. 2008;103(10):2613-2623.

24. Akamizu T, Iwakura H, Ariyasu H, et al. Repeated administration of ghrelin to patients with functional dyspepsia: its effects on food intake and appetite. Eur J Endocrinol. 2008;158(4):491-498.

25. Shinomiya T, Fukunaga M, Akamizu T, et al. Plasma acylated ghrelin levels correlate with subjective symptoms of functional dyspepsia in female patients. Scand J Gastroenterol. 2005;40(6):648-653.

26. Isomoto $\mathrm{H}$, Ueno $\mathrm{H}$, Nishi $\mathrm{Y}$, et al. Circulating ghrelin levels in patients with various upper gastrointestinal diseases. Dig Dis Sci. 2005; 50(5):833-838

27. Kido T, Nakai Y, Kase Y, et al. Effects of rikkunshi-to, a traditional Japanese medicine, on the delay of gastric emptying induced by N(G)nitro-L-arginine. J Pharmacol Sci. 2005;98(2):161-167.

28. Matsumura T, Arai M, Yonemitsu Y, et al. The traditional Japanese medicine Rikkunshito increases the plasma level of ghrelin in humans and mice. J Gastroenterol. 2010;45(3):300-307.
Clinical and Experimental Gastroenterology

\section{Publish your work in this journal}

Clinical and Experimental Gastroenterology is an international, peerreviewed, open access journal, publishing all aspects of gastroenterology in the clinic and laboratory, including: Pathology, pathophysiology of gastrointestinal disease; Investigation and treatment of gastointestinal disease; Pharmacology of drugs used in the alimentary tract;

\section{Dovepress}

Immunology/genetics/genomics related to gastrointestinal disease. This journal is indexed on CAS. The manuscript management system is completely online and includes a very quick and fair peer-review system. Visit http://www.dovepress.com/testimonials.php to read real quotes from published authors. 